\title{
Polychromatic Diagnostics of the Flame with Hilbert Verification of the Visualized Phase Structure
}

\author{
Yu.N. Dubnishchevi,A,C, V.A. Arbuzov,2,A,C, E.V. Arbuzov3,A,B,C, O.S. Zolotukhina4,A,C, \\ V.V. Lukashov5,A \\ A Kutateladze Institute of Thermophysics of the Siberian Branch of \\ the Russian Academy of Sciences, \\ B Sobolev Institute of Mathematics of the Siberian Branch of \\ the Russian Academy of Sciences \\ C Novosibirsk State Technical University \\ ${ }^{1}$ ORCID: 0000-0001-7874-039X, dubnistchev@itp.nsc.ru \\ 2 ORCID: 0000-0003-2404-326X \\ 3 ORCID: 0000-0001-9488-8650 \\ 4 ORCID: 0000-0003-3486-4459 \\ 5 ORCID: 0000-0001-8178-7607
}

\begin{abstract}
The work is aimed at solving the scientific and practical problem of non-disturbing diagnostics of the phase and temperature fields of reacting jets and flames. The method based on polychromatic Hilbert visualization of the phase optical density fields induced by the temperature field in the studied medium, pixel-by-pixel processing of RAW images recorded by the photomatrix in RGB channels, and Hilbert verification of the results is presented with an example of studying an axisymmetric hydrogen-air diffusion flame. The phase structure of the probing light field in the axial symmetry approximation of the flame under investigation is analyzed using the Abel transform. Iterative selection of radial temperature profiles, adapted Bezier curves, is performed with the subsequent calculation of the spatial structure of the refractive index and phase function. The reconstruction of the temperature field by the example of the study of a hydrogen-air flame is carried out taking into account the diversity of the partial optical properties of the gas mixture in a model consistent with the GladstoneDale dispersion formula. The influence of disturbances in the air surrounding the flame on its axial symmetry is discussed. The criterion for the reliability of the research results is a comparison of the hilbertograms obtained in the experiment and the hilbertograms reconstructed from phase structures induced by temperature fields.
\end{abstract}

Keywords: Optical diagnostics of a flame, hydrogen-air diffusion flame, Hilbert optics.

\section{Introduction}

To date, the focus of scientific research is the problems associated with the stabilization of combustion, the features of heat release in the reacting stream and the formation of harmful emissions in combustion products [1]. An adequate solution to these problems is associated with the development of optical diagnostics of reacting jets and flames with obtaining information on the thermodynamic and structural parameters of the medium under study. Modern methods of spectroscopy LIF and RAMAN provide data on the temperature and chemical composition of the flame [2]. At the same time, methods based on diagnostics of the optical phase density fields of the plume remain in demand. In combination with classical methods of visualization of flows [3] using optical tomography [4], such approaches have great potential. 
The article [5] describes a method for assessing the temperature distribution in an axisymmetric flame using high-contrast stereoscopic photography. Spectral reconstruction of temperature fields using pyrometry of color ratios and interferometric tomography is reported [6]. An example of adapted to the problems of flame research is optical diagnostics based on the methods of Hilbert optics and interferometry in combination with pixel-by-pixel processing of the dynamic structure of visualized phase structures induced by temperature fields [7].

The development of polychromatic Hilbert optics methods in flame diagnostics with reconstruction and verification of the spatial phase and temperature structure is the aim of this work.

\section{Hilbert visualization of the phase structure of the flame}

The diagnostic complex is based on the IAB-451 shadow device [8] with modified modules of light emitter, optical filtering, registration and processing of information.

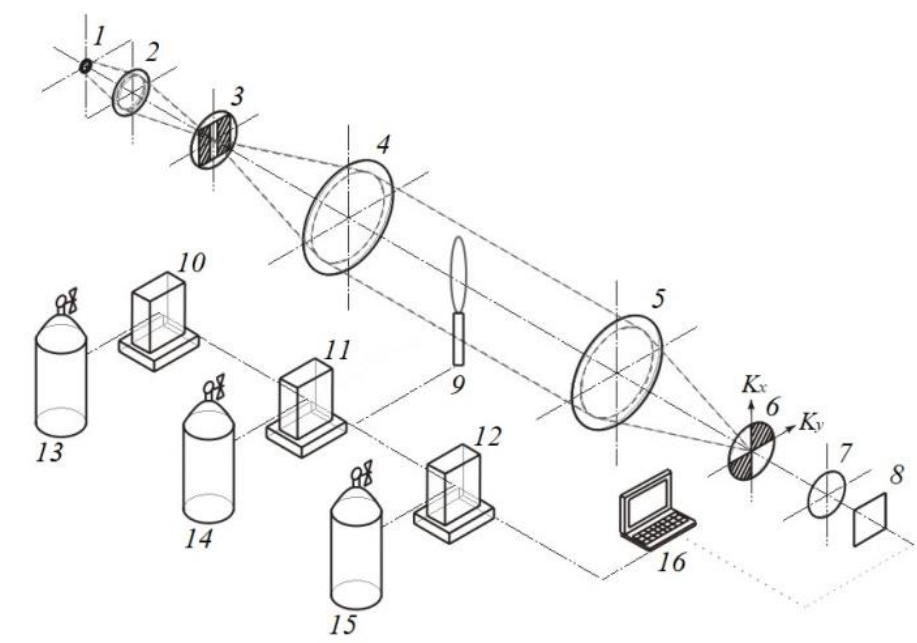

Fig. 1. Experimental setup based on the IAB-451 device.

The experimental complex contains an illumination module consisting of a light source (1) - an RGB LED with operating wavelengths of 636, 537 and $466 \mathrm{~nm}$, a lens (2) and a slit diaphragm (3) located in the front Fourier plane of the objective (4), which forms a probing light field in the medium under study. The Fourier spectrum of phase disturbances induced in the probing field by the torch (9) is localized in the frequency plane of the objective (5), where the Hilbert quadrant filter (6) is located, the orientation of which is matched with the aperture (3). The objective (7) performs an inverse Fourier transform of the filtered field and forms, depending on the spectral characteristics of the light source, an optical analytical signal. This signal is recorded by the photo matrix (8) of the video camera connected to the computer (16).

Directly behind the Hilbert filter (6) in the frequency plane $\left(K_{x}, K_{y}\right)$ for the filtered Fourier spectrum of the light field $S\left(K_{x}, K_{y}\right)$ we have:

$$
H\left(K_{x}, K_{y}\right) s\left(K_{x}, K_{y}\right)=S\left(K_{x}, K_{y}\right) \cos \varphi+\hat{S}_{x}\left(K_{x}, K_{y}\right) \sin \varphi,
$$

where $H\left(K_{x}, K_{y}\right)$ is the transfer-function of the filter, $\hat{S}_{x}\left(K_{x}, K_{y}\right)$ is the Fourier spectrum of the Hilbert-conjugate optical signal:

$$
\begin{gathered}
H\left(K_{x}, K_{y}\right)=\cos \varphi-i \sin \varphi \operatorname{sgn} K_{x}, \\
\hat{S}_{x}\left(K_{x}, K_{y}\right)=-i \operatorname{sgn} K_{x} S\left(K_{x}, K_{y}\right) .
\end{gathered}
$$

Phase shift $\varphi$ is a function of wavelength $\lambda, \varphi=\varphi(\lambda)$.

The objective (7) performs the inverse Fourier transform of the filtered signal:

$$
S\left(K_{x}, K_{y}\right) \cos \varphi+\hat{S}_{x}\left(K_{x}, K_{y}\right) \sin \varphi \leftrightarrow S(x, y) \cos \varphi+\hat{S}_{x}(x, y) \sin \varphi .
$$


Recorded by the photo matrix of the camcorder (8) signal intensity:

$$
I(x, y)=\rho\left\{|S(x, y)|^{2} \cos ^{2} \varphi+\left|\hat{S}_{x}(x, y)\right|^{2} \sin ^{2} \varphi\right\},
$$

where $\rho$ is a sensitivity coefficient of the photo matrix. The Foucault-Hilbert transform is the result of filtering at an arbitrary wavelength $\lambda$ as seen from (1)-(3). The one-dimensional Hilbert transform is performed at the wavelength $\lambda=\lambda_{0}$, satisfying the condition $\varphi\left(\lambda_{0}\right)=\pi / 2$. The Hilbert transform redistributes the signal energy from the low spatial frequency region to the high frequency region.

Fig. 2 shows Hilbert images of a hydrogen-air flame obtained by simultaneous shooting at three different wavelengths of the radiation source $-\lambda_{1}=636 \mathrm{~nm}, \lambda_{2}=537 \mathrm{~nm}$ and $\lambda_{3}=$ $466 \mathrm{~nm}$. The fuel mixture from gas cylinders 13-15 using flow meters 10-12 was fed vertically into stationary air through a tube with an inner diameter of $\mathrm{d}=5 \mathrm{~mm}$ and a length of 500 $\mathrm{mm}$. The tube material is stainless steel. The experiments were carried out in the range of Reynolds numbers $R e=1000 . .2000$, satisfying the Poiseuille laminar flow. The composition and consumption of the fuel mixture of hydrogen with nitrogen was set using a programcontrolled generator of calibration gas mixtures UFPGS-2.
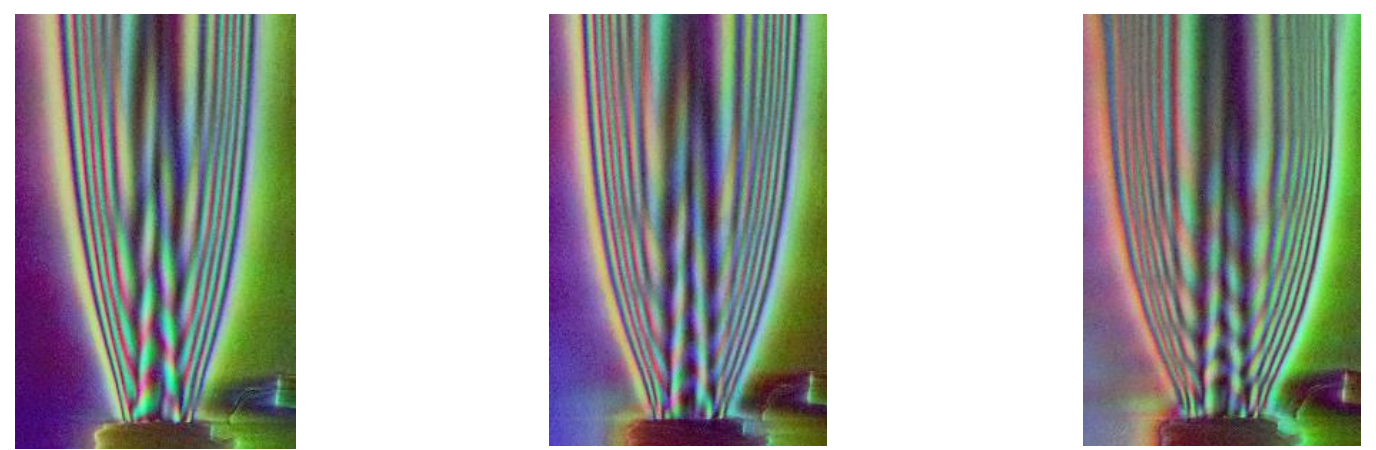

Fig. 2. Hilbert images of hydrogen-air diffusion flames obtained by simultaneous shooting at three different wavelengths of the radiation source $-\lambda_{1}=636 \mathrm{~nm}$, $\lambda_{2}=537 \mathrm{~nm}$ and $\lambda_{3}=466 \mathrm{~nm}$.

Phase structure of the probing light field in diagnostics of an axisymmetric flame is determined in a section with a radius $R$ at a distance $y$ from the end of the tube through the Abel equation:

$$
\Delta \psi(r, y)=2 k \int_{x}^{R}\left[n(r, y)-n_{0}\right] \frac{r d r}{\sqrt{r^{2}-x^{2}}},
$$

where $r^{2}=x^{2}+z^{2} ; k=2 \pi / \lambda$ is the wavenumber; $n(r, y)$ is the refractive index as a function of the distance $r$ from the center of the section; $n_{0}$ is the refractive index of the medium undisturbed by the flame (Fig. 3). $z$ axis is set by the direction of the probing light beam. Coordinates $z_{1}, z_{2}$ limit the size of the flame section in the direction of the probe beam, and $y$ indicates the position of the section along the flame axis.

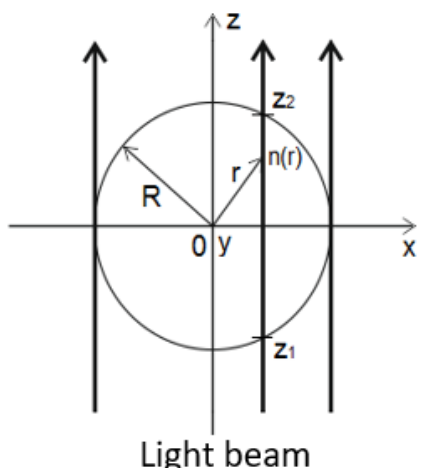

Fig. 3. Flare section in the plane $y=$ const. 


\section{Reconstruction of the radial temperature profile in the structure of a hydrogen-air diffusion flame}

According to the dispersion formula of Gladstone-Dale [9, 10]

$$
n_{k}(\lambda)=A_{k}\left(1+\frac{B_{k}}{\lambda^{2}}+\frac{D_{k}}{\lambda^{4}}\right)
$$

the refractive index of the $k$-th component of the burning mixture depends on the wavelength of the radiation source and parameters $A_{k}, B_{k}$, and $D_{k}$, which are determined empirically by measuring $n_{k}(\lambda)$ for three different values of $\lambda$. When calculating by formula (5), we can restrict ourselves to a term with a quadratic dependence on the wavelength.

The refractive index of the entire mixture (hydrogen-air flame) is defined as

$$
n-1=\frac{p}{p_{\text {n.c. }}} \frac{T_{n . c .}}{T} \sum_{k} A_{k}\left(1+\frac{B_{k}}{\lambda^{2}}\right) \cdot C_{k}
$$

where $p$ is the pressure; $p_{n . c .}-$ atmospheric pressure under normal conditions $(101325$ $\mathrm{Pa}) ; T$ - temperature; $T_{n . c .}$ - temperature under normal conditions $\left(\mathrm{O}^{\circ} \mathrm{C} \sim 273 \mathrm{~K}\right)$.

The method for reconstructing the temperature distribution from the measured values of the refractive index is based on equation (6), which relates $n$ to the temperature and composition of the gas mixture at a point. When hydrocarbons are burned in air, the refractive indices of the reacting components differ slightly from each other. A hydrogen flame, in contrast to a hydrocarbon flame, is characterized by a wide variety of partial optical properties of the gas mixture. In this case, an assessment of the content of the main components $\mathrm{H}_{2}, \mathrm{~N}_{2}, \mathrm{O}_{2}$ and $\mathrm{H}_{2} \mathrm{O}$ is required. The sufficiency of such a partial structure for determining the refractive index of a mixture was verified using experimental data [11]. It should be noted that when working with a hydrogen-oxygen flame, taking ionization into account can affect the accuracy of determining the temperature [12].

An image of the Hilbert-visualized structure of a hydrogen-air flame, obtained synchronously at three different wavelengths $\left(\lambda_{1}=636 \mathrm{~nm}, \lambda_{2}=537 \mathrm{~nm}\right.$ and $\left.\lambda_{3}=466 \mathrm{~nm}\right)$, is shown in Fig. 4.a. The division of the frame into RGB channels is shown in Fig. 4.b-4.d.

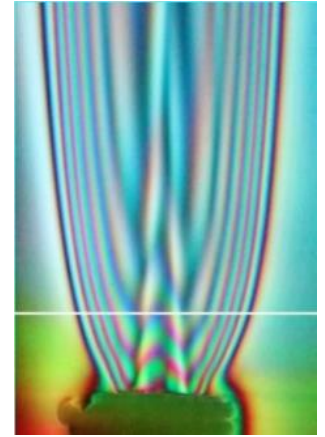

a)

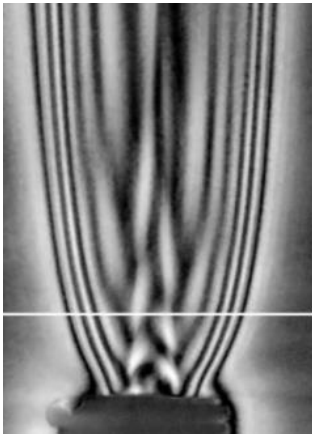

b)

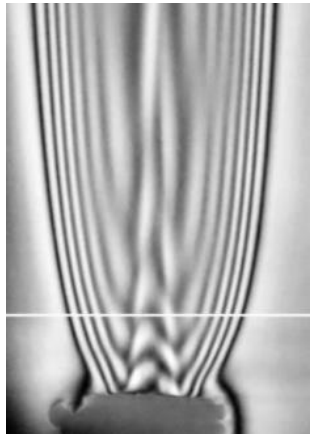

c)

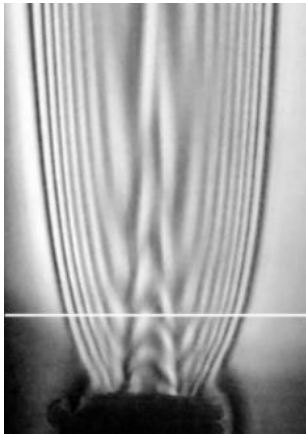

d)

Fig. 4. (a) - RGB-hilbertogram of a hydrogen-air flame $\left(\mathrm{H}_{2} / \mathrm{N}_{2}\right.$, hydrogen volume fraction 25\%); (b) - R-channel, $\lambda_{1}=636 \mathrm{~nm}$; (c) - G-channel, $\lambda_{2}=537 \mathrm{~nm}$; (d) - B-channel, $\lambda_{3}=466 \mathrm{~nm}$; (white line is $6 \mathrm{~mm}$ cross-section from the end of the burner tube).

To solve the inverse problem - to restore the flame temperature $T$ and molar fractions of fuel combustion products $C_{k}$, it is necessary to restore the value of the phase function $\Delta \psi$ from the experimental hilbertogram and, having solved the Abel equation, determine the refractive index $n$ of the medium. Thus, by carrying out measurements at different wavelengths and using formulas (5)-(6), it is possible to restore the values of molar concentrations of fuel combustion products and, accordingly, the temperature distribution in the flame.

The area of combustion of a hydrogen-air flame can be conditionally divided into two zones, consisting of 3 main chemical elements of combustion products. Inner zone $-\mathrm{H}_{2}, \mathrm{H}_{2} \mathrm{O}$ 
and $\mathrm{N}_{2}$; outer zone $\mathrm{O}_{2}, \mathrm{H}_{2} \mathrm{O}$ and $\mathrm{N}_{2}$. The zone boundary is the area with the maximum temperature (flame front), since in its vicinity both the fuel and the oxidizer tend to zero. To determine $T$ and $C_{k}$ in each zone it is sufficient to make measurements at three different wavelengths of the probing field.

In fig. 9 there is a graph illustrating the initial recovery of the phase function $\Delta \psi(r, y)$ at 6 $\mathrm{mm}$ from the end of the burner tube for the R-channel. The red curve represents the phase function $\Delta \psi(r, y)$, the blue curve is the section of the experimental hilbertogram, the black curve is the hilbertogram reconstructed from the phase function $\Delta \psi(r, y)$. o mm is the center of the burner tube.

The method for determining the phase function consisted in iterative sequential selection of the shape and height of the curve represented by the Bezier polynomial (a special case of Bsplines), and the subsequent calculation of the refractive index $n(r, y)$. Further, according to the distribution $n(r, y)$ for the selected area, the hilbertogram is reconstructed, which is compared with the experimental hilbertogram. The iterative algorithm is repeated until the coordinates of the local extrema of the experimental and reconstructed hilbertograms coincide. The coincidence of coordinates of the extrema of the experimental and reconstructed hilbertograms means checking the results obtained. When restoring the phase function $\Delta \psi(r, y)$, the values $A_{k}$ and $B_{k}$ were taken from the reference data.

Fig. 6.a shows the values of the phase function $\Delta \psi(r, y)$ in a $6 \mathrm{~mm}$ section for three RGB channels. Fig. $6 . b$ shows the radial distributions of the refractive index $n(r, y)$, calculated from the obtained data $\Delta \psi(r, y)$.

During the experiment, the pressure in the room was equal to $100500 \mathrm{~Pa}$, the temperature was $24.8^{\circ} \mathrm{C}$, and the air humidity was $19 \%$.
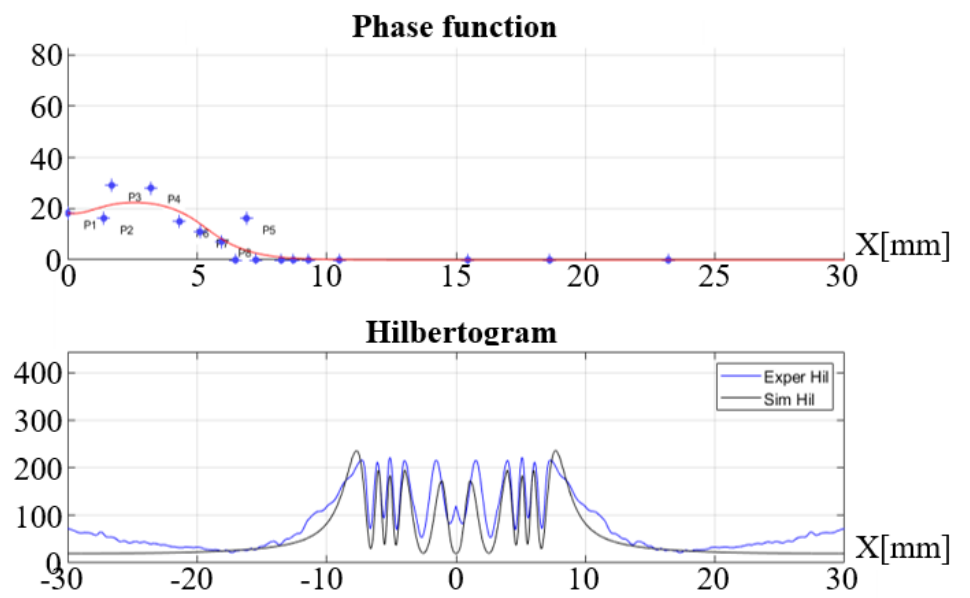

Fig. 5. Experimental and reconstructed phase functions $\Delta \psi(r, y)$ and hilbertograms with a cross section of $6 \mathrm{~mm}$ : R-channel, $\lambda_{1}=636 \mathrm{~nm}$

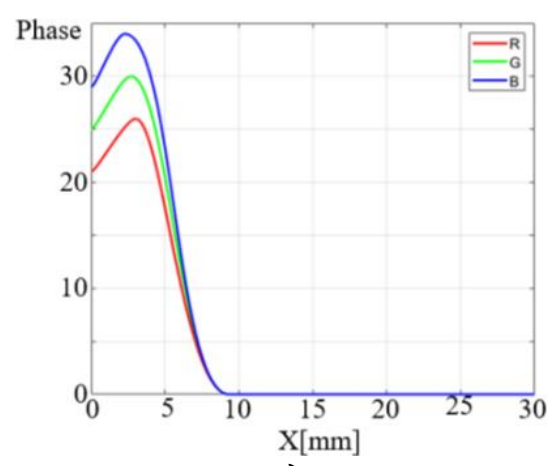

a)

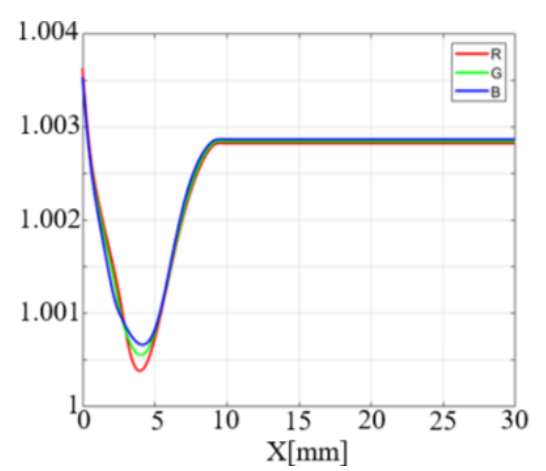

b)

Fig. 6. Section $6 \mathrm{~mm}$ : (a) - reconstructed phase functions $\Delta \psi(r, y)$ for three RGB channels; (b) - reconstructed radial profile of the refractive index $n(r, y)$ for three RGB channels. 
As a verification of the results obtained, the hilbertograms were calculated from the reconstructed phase function and compared with the hilbertograms obtained in the experiment (fig. 7). Comparing the curves, we see that the points of the local minima of the Hilbert bands on the experimental and reconstructed hilbertograms coincide. This confirms the reliability of the approximation of the radial temperature field of a hydrogen-air flame (fig. 8). The discrepancies in the experimental and reconstructed data are due to the violation of the axial symmetry of the flame in a real experiment due to the influence of dynamic disturbances in the air surrounding the flame.

Verification of the results is provided by comparing the experimentally obtained Hilbertograms and those reconstructed from phase structures using the Abel transform. One of the important advantages of the proposed method for flame diagnostics is the potential for measurements with a spatial resolution of up to several microns, which makes it possible to study the entire range of scales of the reacting flow up to Kolmogorov.

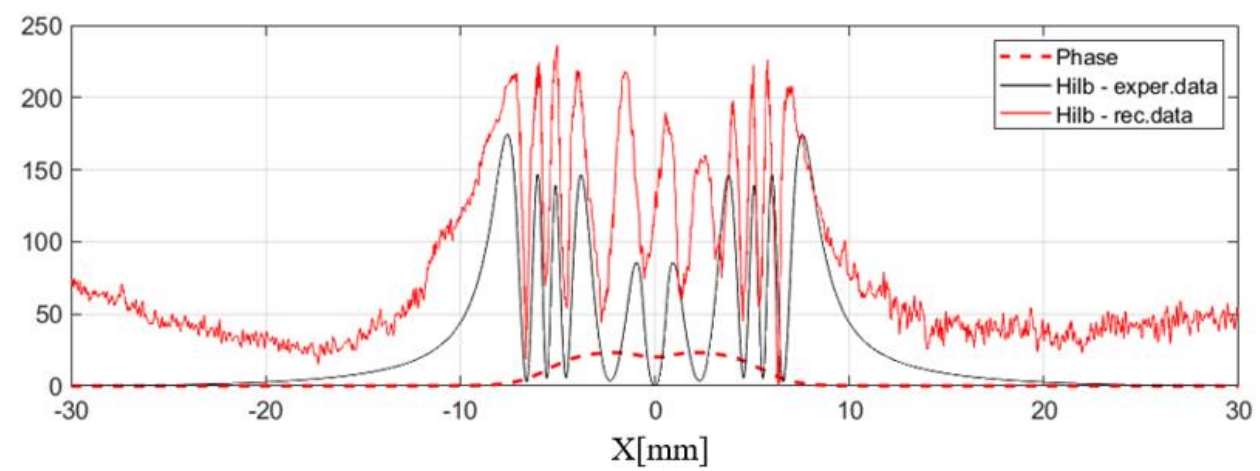

a)

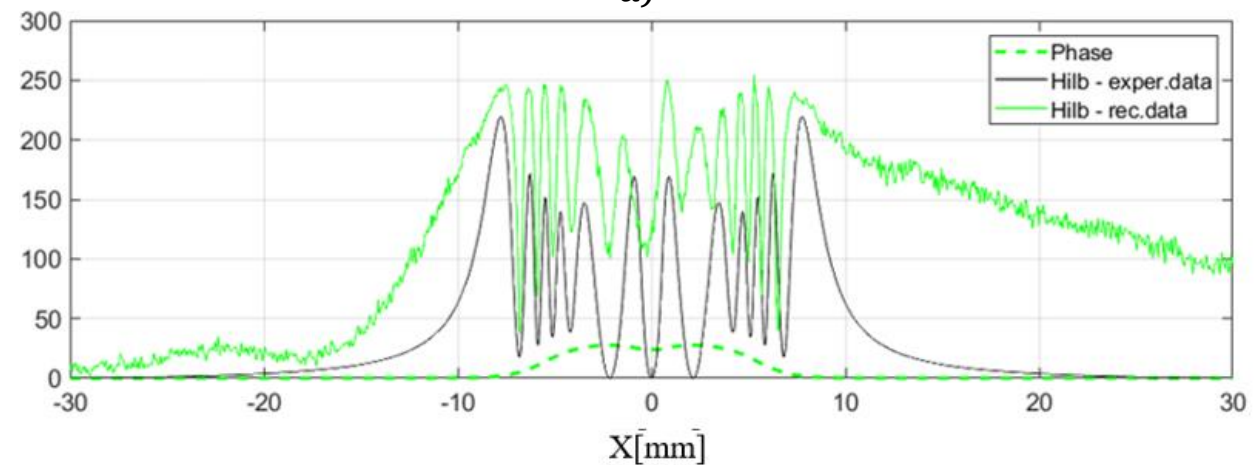

b)

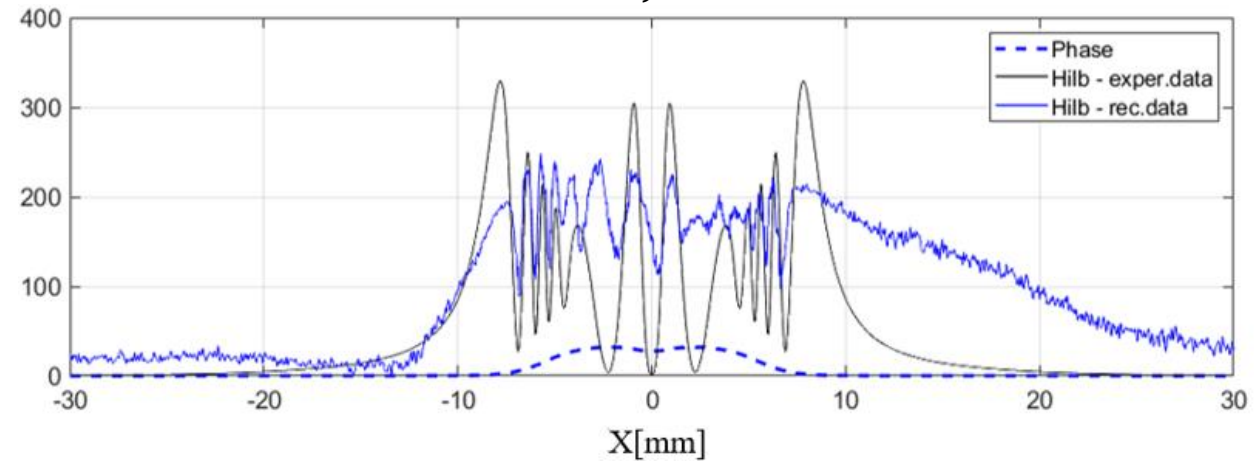

c)

Fig. 7. Comparison of experimental and reconstructed hilbertograms: (a) - R-channel;

(b) - G-channel; (c) - B-channel; the solid line is the section of the experimental

hilbertogram, the solid black line is the reconstructed hilbertogram, the dashed line is the reconstructed phase function $\Delta \psi(r, y)$. 


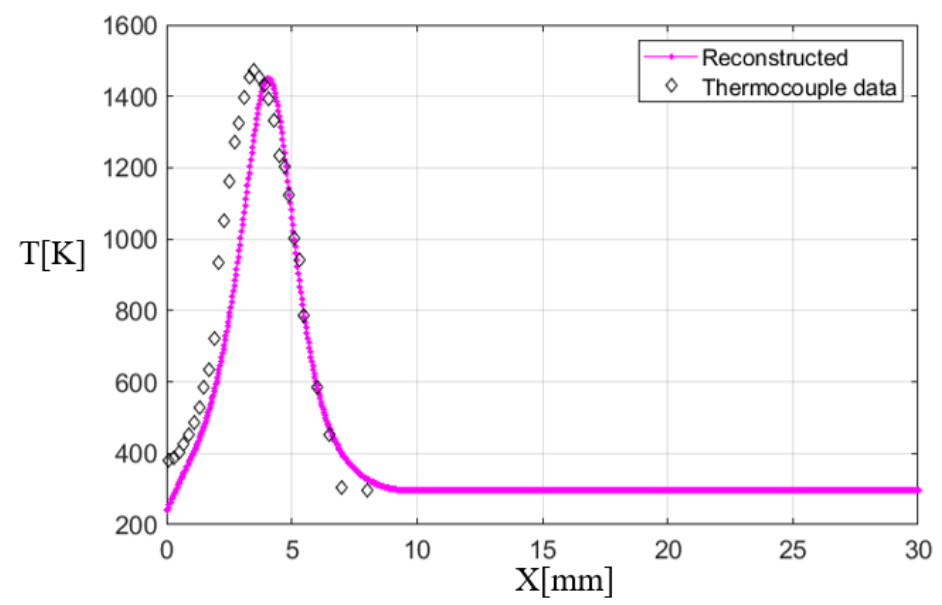

Fig. 8. PeReconstructed radial temperature field of an axisymmetric section of a hydrogen-air flame in a $6 \mathrm{~mm}$ section: solid line - reconstructed temperature $T$; black dots are the temperature measured by the thermocouple.

\section{Conclusion}

The method of polychromatic Hilbert visualization of the phase optical density fields induced by the temperature field in the medium under study is presented by the example of diagnostics of an axisymmetric diffusion hydrogen-air flame. RAW images of the rendered field, registered by the photomatrix in RGB channels, are subjected to pixel-by-pixel processing with Hilbert verification of the results. The phase structure of the probing light field in the approximation of axial symmetry of the flame is analyzed using the Abel transform. Iterative selection of radial temperature profiles, adapted according to Bezier curves, followed by calculation of the spatial structure of the refractive index and phase function is performed. The reconstruction of the temperature field is carried out taking into account the diversity of the partial optical properties of the gas mixture in a model consistent with the Gladstone-Dale dispersion formula. The criterion for the reliability of the results is a comparison of the hilbertograms obtained in the experiment and the hilbertograms reconstructed from the phase structures induced by the temperature field.

The work was carried out within the framework of the state assignment of IT SB RAS No. 121031800217-8.

\section{References}

1. Li J., Huang H., Bai Y., Li S., Kobayashi N. Combustion and heat release characteristics of hydrogen/air diffusion flame on a micro-jet array burner // International Journal of Hydrogen Energy, Vol. 43, №29, 2018, pp. 13563-13574 (doi.org/10.1016/j.ijhydene.2018.04.195).

2. Cheng T. S., Chen J.-Y., Pitz R. W. Raman/LIPF data of temperature and species concentrations in swirling hydrogen jet diffusion flames: conditional analysis and comparison to laminar flamelets // Combustion and Flame, Vol. 186, 2017, pp. 311-324.

3. Smits A. J., Lim T. T. Flow visualization: techniques and example. London: Imperial College Press, 2010.

4. Yong Y., Tian Q., Gang L., Hossain M. M., Gilabert G., Shi L. Recent advances in flame tomography // Chinese Journal of Chemical Engineering, Vol. 20, №2, 2012, pp. 389399 (doi: 10.1016/S1004-9541(12)60402-9).

5. Qunxing Huang, Fei Wang, Jianhua Yan and Yong Chi. Simultaneous estimation of the 3-d soot temperature and volume faction distributions in asymmetric flames using highspeed stereoscopics images // Applied Optics, Vol. 51, №15, 2012, pp. 2968-2978 (doi.org/10.1364/AO.51.002968). 
6. Dreyer J. A. H., Slavchov R. I., Rees E. J. et al. Improved methodology for performing the inverse Abel transform of flame images for cilir ratio pyrometry // Applied Optics, Vol. 58, №10, 2019, pp. 2662-2670 (doi: 10.1364/AO.58.002662).

7. Arbuzov V. A., Arbuzov E. V., Dubnishchev Yu. N., Zolotukhina O. S., Lukashov V. V. Recovery of the temperature field in the case of combustion of premixed propane-air mixtures by methods of the Hilbert optics // Optoelectron., Instrum. Data Process., Vol. 56, №1, 2020, pp. 61-67 (doi: 10.3103/s8756699020010082).

8. Vasiliev L. A. Shadow methods. Publishing house "Science", Main edition of physical and mathematical literature, Moscow, 1968. (in Russian)

9. Hauf V., Grigul U. Optical methods in heat transfer. Mir, Moscow, 1973. (in Russian)

10. Ioffe B. V. Refractometric methods of chemistry, 3rd ed. Rev. L.: Chemistry, Leningrad. separation, 1983. (in Russian)

11. Toro V. V., Mokhov A. V., Levinsky H. B., Smooke M. D. Combined experimental and computational study of laminar, axisymmetric hydrogen-air diffusion flames // Proceedings of the Combustion Institute, Vol. 30, 2005, pp. 485-492 (doi: 10.1016/j.proci.2004.08.221).

12. Yun-yun C., Zhen-hua Li, Yang S., An-zhi H. Extension of the Gladstone-Dale equation for flame flow field diagnosis by optical computerized tomography // Applied Optics, Vol. 48, №13, 2009, pp. 2485-2490 (doi.org/10.1364/AO.48.002485). 